\title{
Use of telephone enquiries to a microbiology laboratory as a proxy measure of reporting efficiency
}

G Phillips, B W Senior, H McEwan

\begin{abstract}
Aims: To assess the efficacy of a bacteriology service in respect of the time interval between collection of specimen and receipt of final report using the number and type of incoming telephone enquiries as a proxy measure.

Methods: For three months, all incoming telephone enquiries regarding the results of bacteriology specimens were monitored. Specimen type, date of sampling, the sender's location and the reason for making the telephone enquiry were recorded.

Results: The number of telephone enquiries made during the study was 1170 , about $5 \%$ of the total number of samples received. Most enquiries related to results of urine cultures. These accounted for $33.9 \%$ of the calls, but only $5 \%$ of the total number of urines cultured. Enquiries relating to blood cultures were the next largest group accounting for $14.9 \%$ of calls, but $11 \%$ of the blood cultures received resulted in a telephone enquiry. The most frequent reason for making the telephone call was that the sender had not received the final report. Conclusions: Clinicians may have unrealistic expectations of the time taken to examine a specimen. A requirement for reporting sterile blood cultures after a shorter incubation period was found, and the value to patient management of earlier reporting of negative urine samples was identified.
\end{abstract}

Optimal patient care means that the interval between the collection of a patient's specimen and the receipt of an accurate report should be as short as possible. Assessing the timeliness of laboratory reports is difficult. Questionnaire surveys have been used ${ }^{1}$ but are time consuming to complete and subject to problems, such as poor response rates and inaccurate completion. Other approaches, such as tracking the specimen from the time of collection to the receipt of the final report by the sender, have also been tried. ${ }^{2}$

This study sought to measure those situations in which results are needed more quickly than the bacteriology laboratory has provided them in a printed form, by investigating all telephone enquiries for results of bacteriological investigations of specimens. The survey was simple and easily undertaken by office staff, required no extra personnel or funding, and had the advantage of being "consumer driven". The aims were to establish which specimen types accounted for most telephone enquiries, which requesting sources were experiencing problems in receiving printed reports, and which factors during the transport of specimens from the patient to the laboratory to the return of the report to the clinician, were the rate limiting steps. It was hoped that the results would also indicate which bacteriological investigations were considered by clinicians to be too prolonged for efficient management of patients and also those investigations for which early communication of interim or negative results could be helpful.

\section{Methods}

COLLECTION OF SPECIMENS AND DELIVERY OF REPORTS

Specimens are collected from general practitioners (GPs) by a hospital van and printed reports are delivered to them by van or post. Delay in receipt of samples coming from GPs in the Dundee area and hospital A was assessed from when the sample was taken and when it arrived in the laboratory. The speed at which printed reports reached GPs from the laboratory was assessed by sending 66 blank forms to 21 practices-11 forms on the Monday, Wednesday, and Friday of two different weeks. These forms were dispatched with a note of explanation in the same envelopes as the routine reports and required only that the receiver note the date and time of receipt of the form and return it to the laboratory in the addressed envelope provided.

Only final authorised results were available to office staff at the laboratory computer terminals for communication to the enquirer. One ward (intensive care unit) also has access to these results via a terminal situated within the ward. No other hospital clinical area or general practice had this facility. Interim results were communicated by telephone only after authorisation by a medical microbiologist.

\section{TELEPHONE ENQUIRIES}

For three months all telephone enquiries about results of bacteriological investigations of specimens were recorded on a standard form by the office staff. The following details were sought from the enquirer: name and date of birth of patient; source of request; and type of specimen and date sent. The following information was also recorded: date and time of enquiry; and the reason for the enquiry which coded as:

A-sender not sure if sample was sent

B-sample not received by laboratory C-no source of request stated on form 
D-sender not received report

E-sender not received report-specimen still under investigation

$\mathrm{F}$-interim results requested

$\mathrm{G}$-further information requested

$\mathrm{H}$-other

The information was entered into an Apple IIe database.

\section{Results}

COLLECTION OF SPECIMENS AND DISPATCH OF REPORTS

It was found that 524 of $566(93 \%)$ samples sent to the laboratory from GPs in the Dundee area arrived in the laboratory within 24 hours of being taken. Of the samples taking two or more days to arrive, 33 of $42(79 \%)$ were taken on a Friday or Saturday and had missed the routine collection. In hospital $\mathrm{A}$, in which the laboratory was situated, 349 of $379(92 \%)$ specimens arrived within 24 hours of being taken. Of those hospital specimens taking two or more days to arrive, 25 of $30(83 \%)$ were taken on a Saturday and missed the routine morning collection service. Overall, delay in receipt of specimens did not seem to be a problem except for those taken in the community on a Friday afternoon and those taken in hospital $A$ at the weekend when there was only one routine collection.

Of the 66 forms dispatched to GPs, $51(77 \%)$ were returned. Only one practice did not return any forms. The results are summarised in table 1. Only $55 \%$ of reports delivered by van and $21 \%$ of those sent by post (38\% overall) were recorded as having been received with 24 hours of issue from the laboratory. Those forms sent out on a Friday seemed to be especially prone to delay. Whether this was because of inefficient delivery or because they were not opened until after the weekend is not known.

Table 1 Time taken for reports of results to reach general practitioners

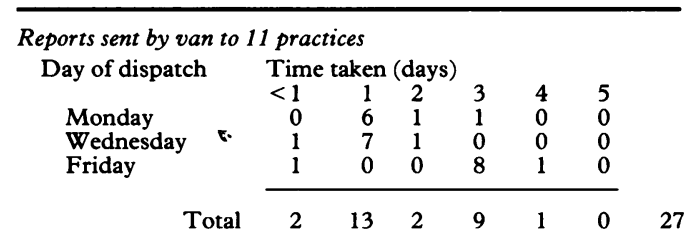

Reports sent by post to 10 practices

Day of dispatch Time taken (days)

\begin{tabular}{|c|c|c|c|c|c|c|}
\hline & & & & & & \\
\hline & $<1$ & 1 & 2 & 3 & & 5 \\
\hline Monday & 0 & 1 & 5 & 1 & c & 0 \\
\hline Wednesday & 0 & 4 & 4 & 0 & c & 0 \\
\hline Friday & 0 & 0 & 1 & 5 & 2 & 1 \\
\hline Total & 0 & 5 & 10 & 6 & 2 & 1 \\
\hline
\end{tabular}

Table 2 Summary of telephone enquiries of specimens

\begin{tabular}{|c|c|c|c|c|}
\hline \multirow[b]{2}{*}{ Specimen group } & \multirow{2}{*}{$\begin{array}{l}\text { Number (\%) } \\
\text { of enquiries }\end{array}$} & \multirow{2}{*}{$\begin{array}{l}\text { Percentage of specimens } \\
\text { in group resulting } \\
\text { in enquiry }\end{array}$} & \multicolumn{2}{|c|}{ Number (\%) of enquiries from: } \\
\hline & & & Hospital $A$ & $G P s$ \\
\hline $\begin{array}{l}\text { Urine culture } \\
\text { Blood culture } \\
\text { Wound swabs } \\
\text { Female genital } \\
\text { Other }^{\star}\end{array}$ & $\begin{array}{c}396(33.9) \\
175(14 \cdot 9) \\
168(14 \cdot 4) \\
66(5 \cdot 6) \\
365(31 \cdot 2)\end{array}$ & $\begin{array}{r}5 \\
11 \\
6 \\
4\end{array}$ & $\begin{array}{r}132(33 \cdot 3) \\
144(82 \cdot 3) \\
62(36 \cdot 9) \\
22(33 \cdot 3)\end{array}$ & $\begin{array}{c}156(39 \cdot 4) \\
0 \\
56(33 \cdot 3) \\
33(50 \cdot 0)\end{array}$ \\
\hline
\end{tabular}

^Included faeces, respiratory tract, body fluid and biopsy specimens, fungal and tuberculosis cultures, antibiotic and C-reactive protein assays.
TELEPHONE ENQUIRIES

The number of telephone enquiries made during the study period was 1170 . This is about $5 \%$ of the total number of specimens submitted for bacteriological examination or antibiotic assay. The results are summarised in table 2 . From this it was apparent that two specimen groups, blood and urine cultures, accounted for most enquiries, and it was to these that further work was directed.

\section{Blood cultures}

Blood cultures were processed by the Bactec NR 730 machine (Becton Dickinson, USA). After six hours' incubation the blood culture bottles are examined twice a day during the first 48 hours' incubation and, if sterile, again on the fourth and the seventh day. Any blood culture giving a positive reading on the machine is Gram stained and subcultured on to solid media for identification and sensitivity testing. It is our routine practice that the clinician is immediately informed by telephone of all Gram stain results. Reports of no growth after 48 hours' incubation are dispatched with a comment that if growth occurs later a further report will be issued. During the study, $9 \%$ of the blood cultures were infected and $96 \%$ of these were identified by Gram stain within 36 hours of receipt in the laboratory.

The results of telephone enquiries about blood cultures from the largest referring source (hospital A) are summarised in table 3. Telephone requests were received for information about $11 \%$ of the blood cultures taken during the study period. A single ward (X) accounted for $52 \%$ of these.

Most $(75 \%)$ enquiries came within three to four days of the sample being taken, with almost half of these coming while the sample was still under investigation.

\section{Urine cultures}

Urine culture in this laboratory is performed using dipslides (Medical Wire \& Equipment Co. Ltd., England), inoculated at the time of sampling. After receipt and overnight incubation in the laboratory sterile urines and those with irrelevant or mixed growths are reported immediately. The bacteria on dipslides with clinically important growth are identified and antibiotic sensitivity tests are set up. The results are read and reported the following day. Thus the minimum turnround time for a negative specimen is 24 hours and for a positive one 48 hours.

The results of telephone enquiries about urine cultures from the two biggest referring sources, hospital A and GPs, are summarised in table 4 . The most frequent reason $(60-74 \%)$ given for the enquiry from both sources was that the report had not been received. A major difference between the two sources was that enquiries tended to come at an earlier stage from hospital A than from GPs.

Table 4 shows that half of the enquiries from hospital A were being received before a positive result was available on the computer; those from GPs were coming at a time when they should have been in receipt of the printed report. 
Table 3 Enquiries about blood culture results from hospital $A$

\begin{tabular}{ll}
\hline $\begin{array}{l}\text { Cumulative number (\%) } \\
\text { of enquiries }\end{array}$ & $\begin{array}{l}\text { Time (days) between sampling } \\
\text { and enquiry } \\
\text { (range }<1-10 \text { days) }\end{array}$ \\
\hline $17(12.5)$ & $\leqslant 1$ \\
$57(41.9)$ & 2 \\
$97(71 \cdot 0)$ & 3 \\
$114(83.8)$ & 4 \\
$127(93 \cdot 4)$ & 5 \\
Commonest reasons for & \\
enquiry: & \\
$\quad$ Not received report- & \\
sample still under & investigation \\
Not received report & $69(47.9 \%)$ \\
\hline
\end{tabular}

^Eight samples did not state date of sampling.

\section{Discussion}

The analysis of the number and nature of telephone enquiries for the results of bacteriological investigations highlighted several important matters. Excepting weekends, specimens were received by the laboratory and the reports received by the clinicians without undue delay. Despite this, telephone enquiries were received for about one in every 20 specimens sent to the laboratory. Most of these concerned blood and urine cultures.

Blood culture enquiries were unacceptably high, being received more frequently than one enquiry for every 10 blood culture specimens submitted. As most of these were made before the initial period of investigation was finished, the clinician was instructed either to telephone again later when a result might be available, or to telephone the on-call microbiologist for an interim report. Many clinical medical staff seemed to be unaware that positive blood culture results were phoned immediately. The provision in the local laboratory user's handbook of information about minimum anticipated turnround times might be helpful and reduce the need to make telephone enquiries.

The reporting of sterile blood cultures after the third rather than the fourth examination on the Bactec NR 730 machine would mean earlier availability of results on the office computer

Table 4 Enquiries about urine culture results from general practices and hospital $A$

\begin{tabular}{|c|c|c|c|}
\hline \multirow{3}{*}{$\begin{array}{l}\text { GPs } \\
\begin{array}{l}\text { Cumulative } \\
\text { number }\end{array}\end{array}$} & \multirow{3}{*}{$\begin{array}{l}\text { Hospital } A \\
\begin{array}{l}\text { (\%) of } \\
\text { enquiries }\end{array}\end{array}$} & GPs & \multirow{2}{*}{$\begin{array}{l}\text { Hospital } A \\
\text { ampling and enquiry }\end{array}$} \\
\hline & & Time (days) between sampling and enquiry & \\
\hline & & (range $\leqslant 1-68)^{\star}$ & (range $\leqslant 1-24) \dagger$ \\
\hline $\begin{array}{c}11(7 \cdot 3) \\
33(21 \cdot 2) \\
49(32 \cdot 7) \\
68(45 \cdot 3) \\
89(59 \cdot 3) \\
105(70 \cdot 0) \\
121(80 \cdot 7)\end{array}$ & $\begin{array}{r}29(22.5) \\
70(54 \cdot 3) \\
94(72 \cdot 9) \\
110(85 \cdot 3) \\
117(90 \cdot 6) \\
118(90 \cdot 7) \\
123(95 \cdot 3)\end{array}$ & & $\begin{array}{r}<1 \\
2 \\
3 \\
4 \\
5 \\
6 \\
7\end{array}$ \\
\hline \multicolumn{2}{|c|}{$\begin{array}{l}\text { Commonest reasons for enquiry: } \\
\text { Not received report } \\
\text { Sample not received by laboratory } \\
\text { Not received report-sample still under } \\
\text { investigation }\end{array}$} & $\begin{array}{r}115(73 \cdot 7 \%) \\
19(12 \cdot 2 \%)\end{array}$ & $\begin{array}{l}80(61 \cdot 1 \%) \ddagger \\
31(23 \cdot 7 \%)\end{array}$ \\
\hline
\end{tabular}

« Six did not state day of sampling.

†Three did not state day of sampling.

†One did not state reason for enquiry. terminals and earlier receipt of reports by the clinician. As $96 \%$ of the positive cultures were identified by the third examination, there would only rarely be need for the issue of an additional report.

Although enquiries for results of urine cultures accounted for the largest number of telephone calls, only $5 \%$ of all the urine cultures submitted to the laboratory led to such an enquiry. Most urine cultures are negative and a ward or clinic screening test for this would be useful if the clinician wished simply to exclude the presence of urinary tract infection. In hospital $\mathrm{A}$, there seemed to be an unrealistic expectation of how quickly culture and antibiotic sensitivity results would be available, but few of these callers actually requested interim results if the final result was unavailable. With the present method of processing urine samples reports cannot be generated faster.

Microbiologists made daily visits to wards like the intensive care unit. Consequently very few telephone enquiries were received from this ward. As a result of identifying ward $X$ as the source of many telephone enquiries for culture results, more regular contact has been made with this ward and the number of enquiries has been reduced.

It is often difficult to decide from the clinical information given on a request form how urgently the result is required. Certain tests are batched in the laboratory for convenience and cost reasons. Moreover, microbiologists and clinicians probably have differing views on the prioritising of specimens. Positive results which are considered important by the microbiologist are likely to be communicated to the clinician immediately, but negative results are not, even though they may be valuable to the clinician for patient management. The use made of interim reports varies among laboratories, but there is a possible need for these to be issued. The laboratory should decide on the minimum acceptable turnround times for each specimen group, and if difficulties are encountered in identification or sensitivity testing an interim report should be issued.

The installation of facilities for direct user access to authorised reports would be helpful to both the clinician and the laboratory. In a questionnaire survey of users' attitudes to their laboratory, however, Pedlar and Bint found that $18 \%$ of those medical staff responding saw this facility as being of very little or no use. ${ }^{1}$ The use of electronic mail might also be helpful, but it is an expensive service and does not overcome the problem of mislaid results if the final filing system is inefficient.

The reasons for making telephone enquiries are varied. They may not always arise because the laboratory or the delivery service has failed to dispatch a report-for instance, results may be mislaid by the receiver. It may also be quicker for a receptionist to telephone for a result than to search for a report which is not in the patient's notes. The experience of many of us is that telephoning within a large hospital can be time consuming and frustrating. Alth- 
ough the use of computer storage of investigations has speeded up the time taken to find a result, certainly for office staff, answering the telephone disrupts other important work and is seen as a wasted duplication of effort when a written report has already been issued. Furthermore, routine enquiries of results already dispatched may prevent other important calls from being dealt with quickly. The changes in practice which are suggested as a result of this survey may help alleviate some of these problems and aid patient care.

We thank Mrs Dorothy Ross and the office staff for their cooperation in this study.

1 Pedlar SJ, Bint AJ. Survey of users' attitudes to their local microbiology laboratory. J Clin Pathol 1991;44:6-9.

2 Rogers S, Bywater MJ, Reeves DS. Audit of turnround times in a microbiology laboratory. J Clin Pathol $1991 ; 44: 257-8$. 